\title{
Relaunch of PAGES Databoard activities
}

ISABELLE LAROCQUE

Institut National de Recherche Scientifique, Centre Eau, Terre et Environnement Québec, Canada; isabelle_larocque@inrs-ete.uquebec.ca

Submitting published data to data centers should be part of the ethics of paleoscience. Data centers have the mandate to collect, organize and provide data to users, but they face reluctance from some scientists to submit their published data. One of the major tasks of the PAGES Databoard, launched in March 2002 (see box), is to "establish a protocol to define the expected flow of data from scientists". Four years after this launch, there is still a lack of engagement from individual scientists from the paleo-community in making their data available. The PAGES SSC recently voted for more prominent actions within the PAGES Databoard. In the new structure of PAGES, data management will become a cross-cutting theme. PAGES thereby aims to reinforce the submission of data from scientists working within PAGES Foci and beyond.

\section{(2) PAGES Databoard}

The PAGES Databoard consists of members from all of the major data archives such as NOAA, PANGAEA and MEDIAS-France as well as from thematic data collection efforts such as the African, European and North American Pollen databases and the IMAGES program. Membership in the PAGES Databoard is open to all interested scientists and organizations.

\section{Goals of the Databoard are to:}

1) establish networks for data management,

2) provide tools to facilitate data contribution,

3) increase data sharing through a common format for data and metadata interchange.

The success of this initiative depends on the cooperation of various database managers but most importantly, it depends on the willingness of individual scientists to submit their data to a participating archive.

\section{Agenda}

First meeting and launch of the initiative: Kandersteg, Switzerland, 4-6 March 2002 Second meeting (planned, closed):

Bern, May 2006

Open meeting: end 2006, early 2007
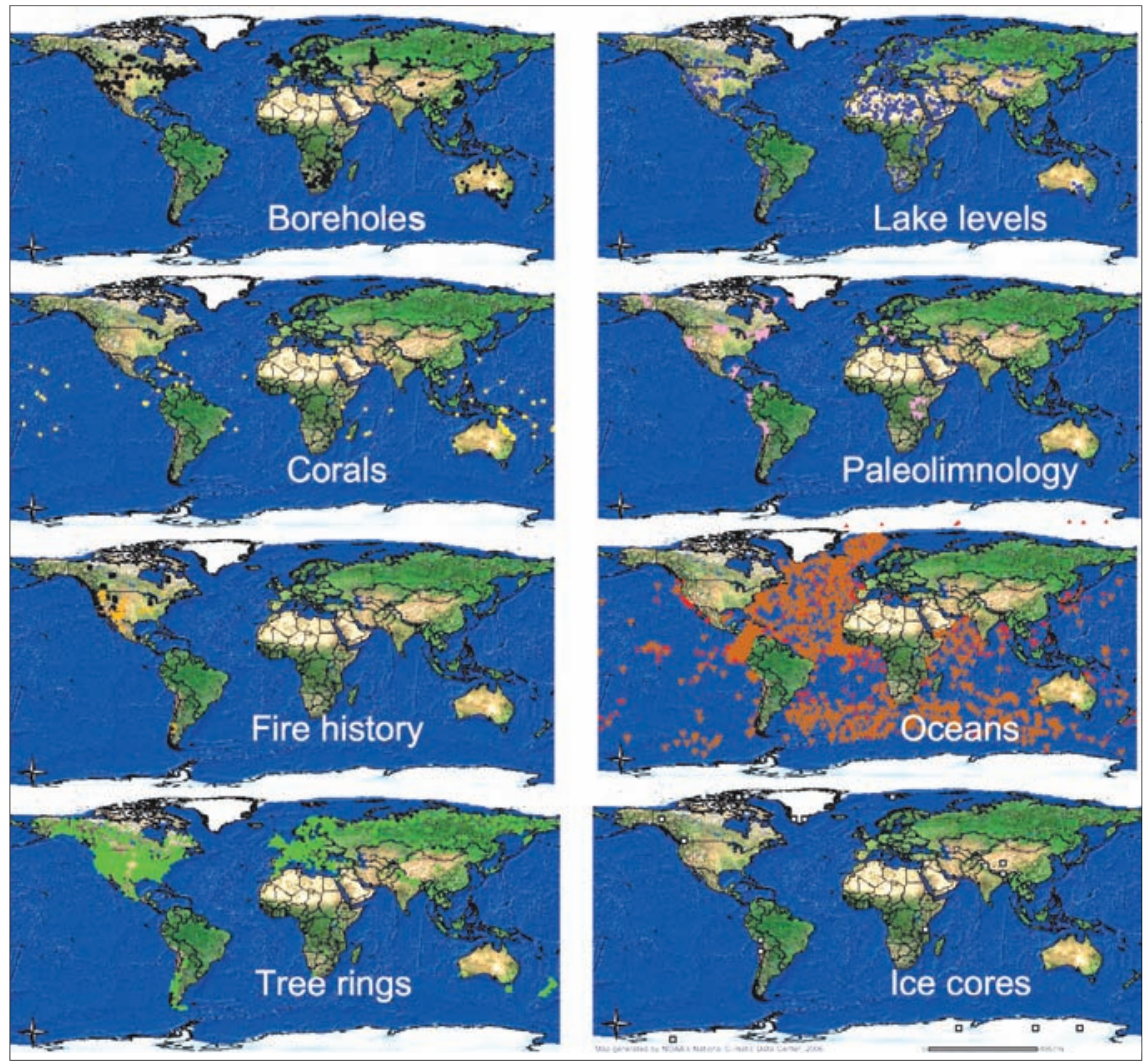

Figure: Maps of the data, divided by paleoarchives, available at the NOAA paleo-data center. The maps were created using free software available on the NOAA website

To reach their goals, data centers need data from around the world, which is far from the case in various paleoresearch fields (see figure). Although many funding agencies have created policies that couple further funding to the submission of data from previous projects, PAGES will adopt a strategy based on science ethics. Every scientist should be proactive in helping to better understand the climate system by submitting his or her individual data to a data center. The more data there is freely available, the easier it is to compare sites and proxies, and to synthesize research results at the local, regional and global scale.

In the new PAGES structure, modeling is emphasized as an integral approach across all Foci, but very few data centers contain modeling data, either input datasets or results from model simulations. This issue will also be targeted by the PAGES Databoard.
The PAGES Databoard will meet in the middle of this year to discuss ways to increase the flow of data to data centers, and to promote a web portal that is user friendly and provides online access to all available data around the world using simple keywords. A list of data centers will soon be updated on the PAGES website. In the following months, PAGES members will be approached in various ways with requests to submit their data to data centers. We hope that by reminding scientists how important data availability is, the PAGES Databoard will strengthen the data-sharing ethic.

If you want to be part of the PAGES Databoard or you want more details on how and where to submit data, please contact Isabelle Larocque at isabelle_ larocque@inrs-ete.uquebec.ca. 